Service social

\title{
Conjoncture actuelle et approches intégrées
}

\section{René Auclair}

Volume 36, numéro 2-3, 1987

Approches intégrées

URI : https://id.erudit.org/iderudit/706359ar

DOI : https://doi.org/10.7202/706359ar

Aller au sommaire du numéro

Éditeur(s)

École de service social de l'Université Laval

ISSN

1708-1734 (numérique)

Découvrir la revue

Citer ce document

Auclair, R. (1987). Conjoncture actuelle et approches intégrées. Service social, 36(2-3), 207-212. https://doi.org/10.7202/706359ar d'utilisation que vous pouvez consulter en ligne.

https://apropos.erudit.org/fr/usagers/politique-dutilisation/ 


\section{AVANT-PROPOS}

\section{Conjoncture actuelle et approches intégrées}

Parler d'approches intégrées dans la conjoncture actuelle où la sécurité sociale et les services sociaux publics sont questionnés, c'est s'interroger face au désordre apparent du monde contemporain et à l'éclatement des modèles de pratique sociale. La nouvelle vision de la réalité proposée maintenant repose sur une approche holistique des phénomènes, c'est-à-dire globale et non fractionnée. Penser globalement, agir localement est une clé majeure de cette vision d'ensemble.

En sciences sociales tout comme en sciences naturelles, les nouveaux concepts ont provoqué une modification profonde de notre façon de voir le monde; de la conception mécaniste de Descartes et Newton, nous sommes passés à une vision holistique et écologique. Le terme " holistique " vient du grec "holos " (tout, entier); il fait référence à une compréhension de la réalité en termes d'un tout intégré dont les propriétés ne peuvent être réduites à des unités plus petites.

Notre société, dans son ensemble, traverse actuellement une crise profonde. Nous en trouvons, chaque jour, de nombreux exemples dans nos quotidiens. Nous connaissons un taux de chômage élevé, nous vivons une crise de l'énergie et de la médecine, nous souffrons de la pollution et d'autres désastres dans notre environnement, nous assistons à une vague montante de la violence et des crimes, etc. Nous vivons, aujourd'hui, dans un monde où tout est intimement imbriqué, un monde dans lequel les phénomènes biologiques, psychologiques, sociaux et environnementaux sont interdépendants. Pour le décrire de manière appropriée, il faut disposer d'une perspective écologique que la vision cartésienne du monde ne nous offre pas.

Nous avons donc besoin, selon Capra ${ }^{1}$, d'un nouveau " paradigme " ou d'une vision différente de la réalité : une "nouvelle grille», une modification fondamentale de notre système de pensée, de nos perceptions et de nos valeurs. Les premiers signes de ce changement, 
de ce passage d'une conception mécaniste de la réalité à une conception holistique, sont déjà perceptibles dans tous les domaines. Les années soixante et soixante-dix ont engendré une série de mouvements sociaux qui semblent tous aller dans la même direction, mettant l'accent sur différents aspects de la nouvelle vision de la réalité. Celle-ci inclut la conception intégrée de la vie, de l'esprit, de la conscience et de l'évolution, la notion holistique qui lui fait pendant dans le domaine de la santé et des services sociaux; l'intégration des approches occidentale et orientale de la psychologie et de la psychothérapie; un cadre conceptuel nouveau pour l'économie et une perspective écologique et féministe de nature fondamentalement spirituelle.

Cette vision d'ensemble de la vie est une base appropriée non seulement pour les sciences naturelles et du comportement, mais aussi pour les sciences sociales et, en particulier, pour le travail social.

L'oscillation entre une approche parcellaire et une approche intégrée des problèmes sociaux est une constante dans l'histoire du service social. En effet, selon les époques, la nature des problèmes sociaux a fait appel soit à un changement global, soit à plusieurs expertises qui risquaient non seulement d'opposer les solutions, mais aussi de faire éclater la profession. La profusion actuelle des modèles de pratique en service social au Québec et aux États-Unis illustre bien ce phénomène.

La nouvelle vision de la réalité dont nous venons de parler se fonde sur une conscience de l'interdépendance essentielle de tous les phénomènes physiques, biologiques, psychologiques, sociaux et culturels. Elle transcende les limites disciplinaires et conceptuelles, actuelles. Pour l'instant, il n'existe pas de cadre bien défini, conceptuel ou institutionnel, qui conviendrait à la formulation du nouveau paradigme, mais de nombreux individus, communautés, réseaux, élaborent de nouveaux modes de pensée, s'organisent en fonction de nouveaux principes et étudient déjà le profil que devrait présenter un tel cadre.

\section{Buts}

Les buts que nous visons dans ce numéro à thème sont triples. Premièrement, nous voulons acquérir des connaissances plus précises sur les concepts théoriques d'un mode d'approche intégré. Deuxièmement, nous voulons approfondir avec les intervenants leur pratique professionnelle en tentant d'identifier et d'analyser avec eux les modes d'agir qui structurent déjà leur façon d'intervenir. Et, troisièmement, nous voulons identifier de façon précise les zones de progrès à réaliser dans ce domaine. 


\section{Approche(s) intégrée(s) : essai de définition}

Pincus et Minahan ${ }^{2}$ affirment qu'il ne devrait pas exister de division du travail, c'est-à-dire d'une part offrir des services afin de répondre aux besoins individuels et d'autre part, rechercher des solutions aux enjeux collectifs. Plusieurs problèmes personnels ne peuvent être solutionnés par une personne ou une famille parce qu'ils sont reliés à des enjeux collectifs. À l'inverse, ces derniers ne peuvent être résolus sans tenir compte de leur impact sur les problèmes individuels.

Le mode d'approche intégré que plusieurs universités américaines expérimentent veut promouvoir une vision globale holistique des différentes responsabilités que doit assumer le travailleur social. "L'approche intégrée que nous recherchons peut être observée, nous l'espérons dans une vision holistique de la gamme normale des responsabilités du travailleur social. » ${ }^{3}$

Or, ces responsabilités impliquent des interventions cliniques (auprès d'individus, de familles ou de groupes), de même qu'auprès des organismes et des communautés.

Ce mode d'approche se définit comme plus réaliste que l'approche traditionnelle. D'ailleurs, le modèle traditionnel est centré sur la méthode alors que le modèle intégré est centré sur le problème. Il se veut plus réaliste, parce qu'il est absolument nécessaire d'avoir une vision d'ensemble d'un problème social avant de le subdiviser. Les causes des problèmes sociaux telles que définies sont complexes et sont reliées à des facteurs d'ordre individuel, familial, aux groupes, aux organismes et aux communautés. Cette interrelation des différents facteurs doit donc être reconnue non seulement au plan de la définition des problèmes mais aussi au niveau des moyens utilisés pour les réduire.

Il existe différentes orientations ou perspectives théoriques ayant permis le développement de divers modes d'approche intégrée. Pour sa part, Garvin s'est attardé à comparer les différentes approches pour une pratique généraliste. Ceci l'a amené à une classification représentée sous trois vocables, selon les principales théories de base qui ont guidé les auteurs; il s'agit de la perspective centrée sur la tâche, de la perspective systémique et enfin, de la perspective psychosociale ${ }^{4}$.

De leur côté, Moreau, Lévesque et Panet-Raymond établissent une distinction entre les approches intégrées consensuelles et conflictuelles. Ils proposent une approche structurelle qui essaie de tenir compte des rapports inégalitaires qui existent entre les différents systèmes en présence dans une situation-problème. Cette approche accorde une grande importance à l'influence des facteurs structurels telles les conditions de vie et de travail sur les comportements personnels et interpersonnels ${ }^{5}$. 
Malgré l'intérêt que l'on puisse porter au mode d'approche intégrée, que l'on soit intervenant ou bénéficiaire d'une intervention de ce type, quelques auteurs ont relevé un certain nombre d'interrogations portant sur des éléments bien précis tels le pouvoir et l'autorité du travailleur social, l'état des connaissances en travail social et le développement du travail par équipe. On notera aussi qu'à l'intérieur de ce numéro, les différents auteurs n'ont pas abordé le concept d'intégration d'une façon univoque ; on retrouve en effet des compréhensions complémentaires, et à des niveaux différents, comme une concertation d'organismes, un travail en équipe, une unification des méthodes d'intervention, une continuité dans l'action, une perspective d'analyse d'ensemble ou socio-politique, une dynamique d'action planifiée ou un idéal paradigmatique.

\section{Présentation}

Dans ce numéro, nous voulons présenter une vue d'ensemble de la situation des approches intégrées en service social, principalement par des réflexions globales sur la signification et la portée de ces approches, pour la société et notre discipline, par le compte rendu de recherches et d'expériences de pratique en cours dans le domaine, tant au Québec qu'à l'extérieur et par un retour sur la formation à l'approche intégrée.

Plus spécifiquement, Laforest montre que, à différentes époques et dans différentes conjonctures, les écrits comme les pratiques se sont avérés porteurs d'un mouvement de recherche de convergence et de synthèse à l'intérieur du travail social. À l'aide de quelques pratiques interreliées, Moreau décrit l'approche structurelle et il en montre les implications concrètes, les techniques d'intervention et les dilemmes qu'elle soulève. Préoccupée par la situation des femmes et par les pratiques, Bourgon décrit à l'aide d'un exemple l'approche féministe en tenant compte de l'état actuel des rapports sociaux. À partir d'études qui associent la pauvreté et les mauvais traitements faits aux enfants, Chamberland, qui s'inspire d'un cadre écologique, montre que la réduction de la violence doit passer par une réorientation profonde des valeurs et des systèmes politiques et économiques. Pour conclure cette partie sur les courants théoriques, Auclair fait une synthèse et un commentaire des écrits sur l'approche intégrée.

La deuxième partie de ce numéro réunit des textes dont le point commun est la description des pratiques de l'approche intégrée, pratiques conceptualisées dans un cadre de recherche en ce qui concerne les deux premiers articles: Auclair et Lampron décrivent, en les resituant dans leur contexte historique et théorique, les principaux éléments de 
l'approche intégrée telle que pratiquée au Centre de services sociaux de Québec, succursale de Charlevoix. À partir de la pratique auprès des cas d'abus d'enfants en Angleterre, Davies montre la nécessité d'une démocratisation des structures du service social et le potentiel d'une mise en commun des situations indiviudelles. David, se fondant sur une expérience de trois ans au Bureau des services sociaux Laval (C.S.S. Montréal métropolitain), soutient la nécessité et l'efficacité d'une approche intégrée dans le traitement de l'inceste. Travailleur social dans un contexte de psychiatrie infantile et juvénile, Néron analyse une approche de prise en charge qui intègre le contrôle social et la thérapie. Décrivant une expérience mettant à contribution différents organismes socio-sanitaires et scolaires, Ouellet et Richard ont voulu démontrer que le travail social peut et doit modifier ses approches et modes d'intervention institutionnels. Desrosiers, enfin, offre une réflexion liée à une expérience portant sur la gestion du stress au travail et vécue par des conseillers syndicaux à Québec.

L'intégration des pratiques s'est aussi manifestée dans les programmes de formation. Lévesque et Panet-Raymond relatent leur expérience d'enseignement d'une approche intégrée à l'École de service social de I'Université de Montréal. À titre d'acteur et de témoin privilégié, Michea fait une présentation descriptive de trois propositions d'approches intégrées et de méthodologies de base qui, dans les années soixantedix, ont été des points marquants dans l'histoire du service social d'Amérique du Sud. Dans un cadre écologique, Bouchard rend compte de la demande d'intervenantes engagées avec lui dans un atelier de formation continue et impliquées auprès de familles faisant l'objet de plaintes pour négligence envers un enfant. L'article de Favreau conclut cette partie et le numéro : partant du contexte des crises d'identité et de légitimité vécues par le travail social dans les deux dernières décennies, il esquisse quelques pistes d'avenir pour la profession et les incidences sur la formation en ce domaine.

École de service social,

René AUCLAIR

Université Laval.

\section{Notes et références}

1 CAPRA, F., Le temps du changement, Monaco, Éditions du Rocher, 1983, pp. 12-15.

2 Pincus, A. et Minahan, A., Social Work Practice: Model and Method, Itasca (III.), Peacock Publishers, 1973. 
${ }^{3}$ Garvin, C., "Education for Generalist Practice : A Comparative Analysis of Current Modalities", Teaching for Competence in the Delivery of Direct Services, New York, Council on Social Work Education, 1976.

${ }^{4}$ Garvin, C., op. cit., p. 19.

5 Moreau, M., J. Lévesque et J. Panet-Raymond, "L'approche structurelle : un défi pour les éducateurs en travail social", La formation en travail social au Québec : d'une école à l'autre, Montréal, Regroupement des unités de formation universitaire en travail social au Québec, Montréal, 1984. 